\title{
Application of Connectionist Semantic Memory Model in Building Q\&A Bank
}

\author{
Noha M. Zakzouk \\ Training Sector, \\ Ministry of Military Production, \\ Cairo, Egypt
}

\author{
Hassan M. Bedour, PhD \\ Computer \& Systems Engineering \\ Dept., \\ Faculty of Engineering, \\ Ain Shams University, Cairo, Egypt
}

\author{
Dina M. Khorshied, PhD \\ Science and Technology Center of \\ Excellence, Ministry of Military \\ Production, Cairo, Egypt
}

\begin{abstract}
Several trials has been made to simulate human's memory with different techniques. This memory has very complicated structure with enormous storage space of data. It has also different memory categories and organizations such as semantic memory. The semantic memory itself has different models according to psycholinguistic view. This paper introduces a novel semantic memory model according to neural fuzzy concept with the use of Fuzzy Inference System (FIS). FIS is used to implement the neural network that simulates the neurons for human's memory stored data structure. The use of fuzzy system combined with neural network (neural-fuzzy) here helps to use a set of IF- THEN rules to either train the data or estimate the answer in the Question/Answering system built. There is a set of (what) questions asked by the user and their answer(s) is (are) estimated by the designed FIS and the software then displayed on a designed Graphical User Interface (GUI). This simulation tries to think as human's brain does. The implementation here is occurred using approximately pulse membership functions as the entered statement will be digitalized in their corresponding order numbers. The defuzzification method is used by Sugeno type that gives constant output and has several advantages. This helps also to give definite answer(s) to the asked question not just an answer probability scale. The GUI displays one or more answer according to the user choice. In all cases, it gives a complete meaningful statement. The simulation results are promising and encouraging.
\end{abstract}

\section{Keywords}

Neural Network; Fuzzy Inference System; Semantic Memory; Connectionist Memory Model

\section{INTRODUCTION}

Semantic computing field is commonly used to address the derivation and matching of semantics, and describes several approaches such as the organization of memory categories [1]. There is a necessary demand for acquisition and representation of semantic concepts to understand the natural languages by cognitive systems [2].

There is an asked question about how the mind/brain represents the words, and sentences meaning. Its answer is provided by analyzing the statistical structure of language to infer the optimal way that may have generated that structure and its representation method [3].

Human's memory allows everyone to capture their information from the life experiences and take advantage of this information in the future. This stored knowledge about the world, including knowledge about people, places, and facts makes up what is known as the semantic memory [4].
This semantic memory is considered a kind of memory appeared on the psychological science, and is necessary for the use of language [5]. It is necessary for everyone to construct meaning from otherwise meaningless words and utterances, to recognize objects, and to interact with the world in a knowledge-based manner [6].

Different psycholinguistic theories of semantic memory form the basis of understanding of natural language concepts. There is a growing demand for Natural language processing (NLP) techniques that provide effective algorithms for search of relevant information in a huge amount of text documents available in machine readable form. It is known that there is no computer system that is able to use the language in the same way as humans do, but there are some implementation techniques help to improve human - computer interfacing [7].

It is a fact that major component of a fuzzy system is set of IF-THEN fuzzy rules representing the relevant knowledge (i.e. fuzzy model) of the underlying data generating process that is being tracked. A popular approach is the use of neural networks to realize the structures of the fuzzy systems. The integrated system is popularly known as a neural-fuzzy system or fuzzy neural network [8].

In this paper, a novel model of the connectionist semantic memory is proposed using fuzzy logic concept, and Fuzzy Inference System (FIS) structure. This proposed model is considered as combination between the neural-network used in the connectionist semantic memory model and the fuzzy logic system properties. This proposed model is used in building question/answer bank.

In section 2 , there is a description of semantic memory models and their different theories. At section 3, the proposed model design and implementation are explained in detail. Finally, section 4 talks about the expected future work and concludes the proposed research work.

\section{SEMANTIC MEMORY MODELS}

Memory has been viewed as a storehouse of items that are created, and then filed away as if they were books in a library. Storage, loss, organization, and retrieval of memories have all been considered from this point of view [9].

Several semantic memory models with their structures, and description theories are discussed later in the following section. They describe how lexical elements are stored and processed by human brains [7]. These models are summarized as:

1. Hierarchal model.

2. Spreading activation model.

3. Connectionist model.

4. Online neural-fuzzy semantic memory model. 


\subsection{Hierarchal model}

This model is stated in [10] as the first model proposed for storing semantic information in a computer memory. Each word is stored by set of pointers to other words in the memory in the form of a tree. This structure helps to construct meaningful relationships using these words. If this structure is used for modeling human memory, this results in testable predictions about retrieving information.

In this model, concepts are stored as nodes in a network, and predicates are stored as labeled links between nodes. According to this theory, if concepts at different levels of specificity were linked with 'is_a' predicates, then the system could provide an economical means of knowledge storage and generalization [11].

\subsection{Spreading activation model}

This model organizes concepts in the form of a lexical network. Searching mechanisms is proposed where the activation of probe concept would spread out along all outgoing links, activating other nodes related to the probe, which in turn could pass activation via their own links [11]. The properties links between nodes increases according to the relations between the two concepts [12].

\subsection{Connectionist model}

This model structure is represented as a neural network. Here the brain is considered as a model of a parallel computational device consists of large richly interconnected network units as described in [13]. The mathematical models used in brain-like systems are simplified and developed. This computational style has large richly interconnected neuron units to form the neural network. This will be similar to the human brain enormous number of processing elements (neurons). Much of the connectionist research on learning can be viewed as an attempt to use statistics in a much richer way by using hierarchical nonlinear models that take higher order statistics into account [14].

Figure 1 illustrates the connectionist model of semantic memory adapted from [15], used to learn all the propositions true of the specific concepts ('pine', 'oak', 'rose', etc.) in the Quillian model [11]. Connections run from a pool of concept units and a pool of relation units to output units for the concepts and for each of the different types of properties ( ' $i s$ ', 'has', and 'can') properties. There are two pools of internal or 'hidden' units, between the input and output. One pool, called concept representation units, sits between the concept units and the other pool, called general hidden units, which receives input from the concept representation units and the relation units [9].

There are some input units on the left and the activation propagates from the left to the right. In this model the input units appear on the left, and activation propagates from the left to the right. Each unit in the Item layer corresponds to an individual object in the environment. Each unit in the Context layer represents contextual constraints on the kind of information to be retrieved. Thus, for 'canary' example, the input pair 'canary' can corresponds to a situation in which the network is shown a picture of a canary, and asked 'what it can $d o$ ?'. The network is trained to turn on all those units that represent correct completions of the input query. In the example shown, the correct units to activate are 'grow', 'move', 'fly', and 'sing' [16].

\subsection{Online Neural-Fuzzy Semantic Memory Model (eFSM)}

The implementation of a fuzzy system with neural network results in neural-fuzzy model. The fuzzy model evolves and adapts its knowledge depending on the dynamics of the underlying environment. The eFSM model has a neuralfuzzy-based architecture with an online data-driven adaptive computing structure constructed via incremental learning. This model is essentially a neural-fuzzy system that consists of several layers of computing nodes [17]. In common this system consists of five layers, as illustrated in Figure 2 such as any neural-fuzzy system. These layers are summarized as: the input layer, the antecedent layer, the rule layer, consequent layer, and the output layer.

The input (output) layer signifies the sensory inputs from (outputs to) the association areas of the neocortex, while the antecedent (consequent) layer functionally corresponds to the encoding (decoding) of memory traces in the entorhinal cortex. The rule-base layer subsequently emulates the relational associative mappings of the memory traces in the substructures of the dentate gyrus and the human hippocampus [18]

To add a new fuzzy rule the rule-generating procedure is run and in turns this procedure ensures the novelty of the rules formed and then supports the merging of highly similar fuzzy sets and the pruning of identical rules that may appear due to the incremental learning process. This model is used to perform lifelong learning and is applicable to more complex real-world problems with dynamic or time-varying characteristics as evaluating the learning and modeling performances for some benchmark applications [17].

\section{PROPOSED MODEL}

This research idea came from the use of fuzzy to implement the neural network of the normal connectionist semantic memory model that illustrated before in Figure 1. This is occurred using FIS that is easier than the use of the complex internal structure of eFSM. As a result there will be new network structure that has multiple inputs and multiple outputs as in Figure 3.

\subsection{Proposed model versus other models}

The proposed model uses the structure of the eFSM model as five layers to implement the connectionist model architecture by FIS structure as a main building unit for the system memory. Also in the eFSM the used membership functions were of Gaussian type but in the proposed model Trapezoidal one is used as it is more similar to pulse function. The defuzzification there was by Mamdani method and the proposed model is by Sugeno method. There are several advantages of the Sugeno Method over Mamdani such as:

1. It is computationally efficient.

2. It works well with optimization and adaptive techniques.

3. It is well suited to mathematical analysis.

The semantic process as searching algorithm in [7] was used as a model of the popular 20-question word game*. In that game one is allowed to ask 20 questions trying to guess the concept the opponent has in mind. The result relies on knowledge about the world that is represented as relations between lexical elements. In their implementation of the game the computer, using knowledge encoded in the form of semantic network, tries to guess the object a human user has in mind. That approach was based on the semantic search 
algorithm implemented in the form of a word game performed by the human user, who modifies the lexical knowledge base as a result of her/his search. The interaction with the program has been limited so far to the answers accepted to the questions generated using the knowledge stored in the system. In that implementation, it is allowed only 'yes', 'no', 'don't know', 'sometimes', and 'frequently answers depending on the stored data in the database.

Q1. Is it classified as Animal, Vegetable or Mineral? Animal, Vegetable, Mineral, Concept, Unknown. Q2. Is it black?

Yes, No, Unknown, Irrelevant, Sometimes, Maybe, Probably, Doubtful, Usually, Depends, Rarely, Partly. Q3. Is it colorful?

Yes, No, Unknown, Irrelevant, Sometimes, Maybe, Probably, Doubtful, Usually, Depends, Rarely, Partly.

*http://www.20q.net/

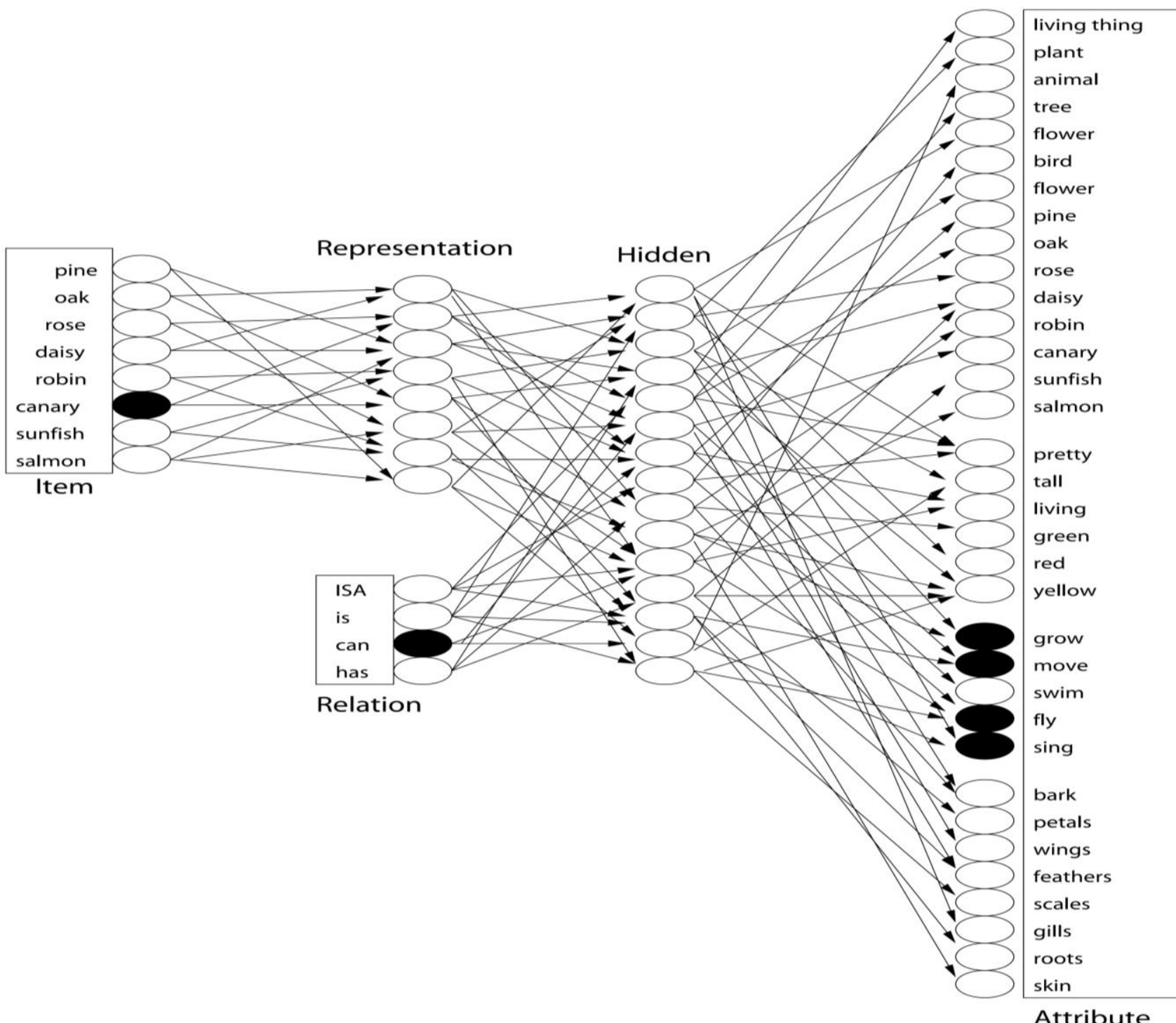

Figure 1. A connectionist model of semantic memory [19]

In the proposed model, a designed Graphical User Interface (GUI) used to form the user query. This GUI is not only give yes/no answer or answer probability scale but also estimates a complete statement. For the same example about item 'canary' there are some different 'what' question stored about 'canary' item such as Table 1.

Table 1. Answers for proposed model

\begin{tabular}{||l||l||l||}
\hline \hline No. & Question? & Answer(s) to be displayed. \\
\hline \hline \multirow{3}{*}{ Q1 } & \multirow{3}{*}{ What is canary? } & Ans1. Canary is a living thing. \\
\cline { 3 - 3 } & & Ans2. Canary is a bird. \\
\cline { 3 - 4 } & & Ans3. Canary is a reptile. \\
\hline \hline Q2 & What can canary do? & Ans1. Canary can grow. \\
\hline
\end{tabular}

\begin{tabular}{|l||l||l|}
\hline \hline \multirow{2}{*}{} & \multicolumn{1}{|l|}{} & Ans2. Canary can move. \\
\cline { 3 - 3 } & & Ans3. Canary can fly. \\
\cline { 3 - 3 } & & Ans4. Canary can sing. \\
\cline { 3 - 3 } & & Ans5. Canary can produce eggs. \\
\hline \hline \multirow{2}{*}{ Q3 } & \multirow{2}{*}{ What does canary have? } & Ans1. Canary has wings. \\
\cline { 3 - 4 } & & Ans2. Canary has feathers. \\
\hline \hline Q4 & What color is canary? & Ans1. Canary is yellow. \\
\hline
\end{tabular}

\subsection{The Graphical User Interface Design}

The GUI is designed to be very user friendly as the user can form his/her question by selecting its parts from several popup menus. These popup menus complete the 'what' question asked, and are summarized as follows: 
1. The first popup menu; is considered the selection of the verb or the auxiliary verb of the question.

2. The second popup menu; is used to select the item for asked question.
3. The third popup menu; is used as either a question end or the main verb of the question according to the rest of user choice to form a meaningful question.

4. The last popup menu; is the multi-answers menu that defines the order of the output answer or the number of the displayed answers.

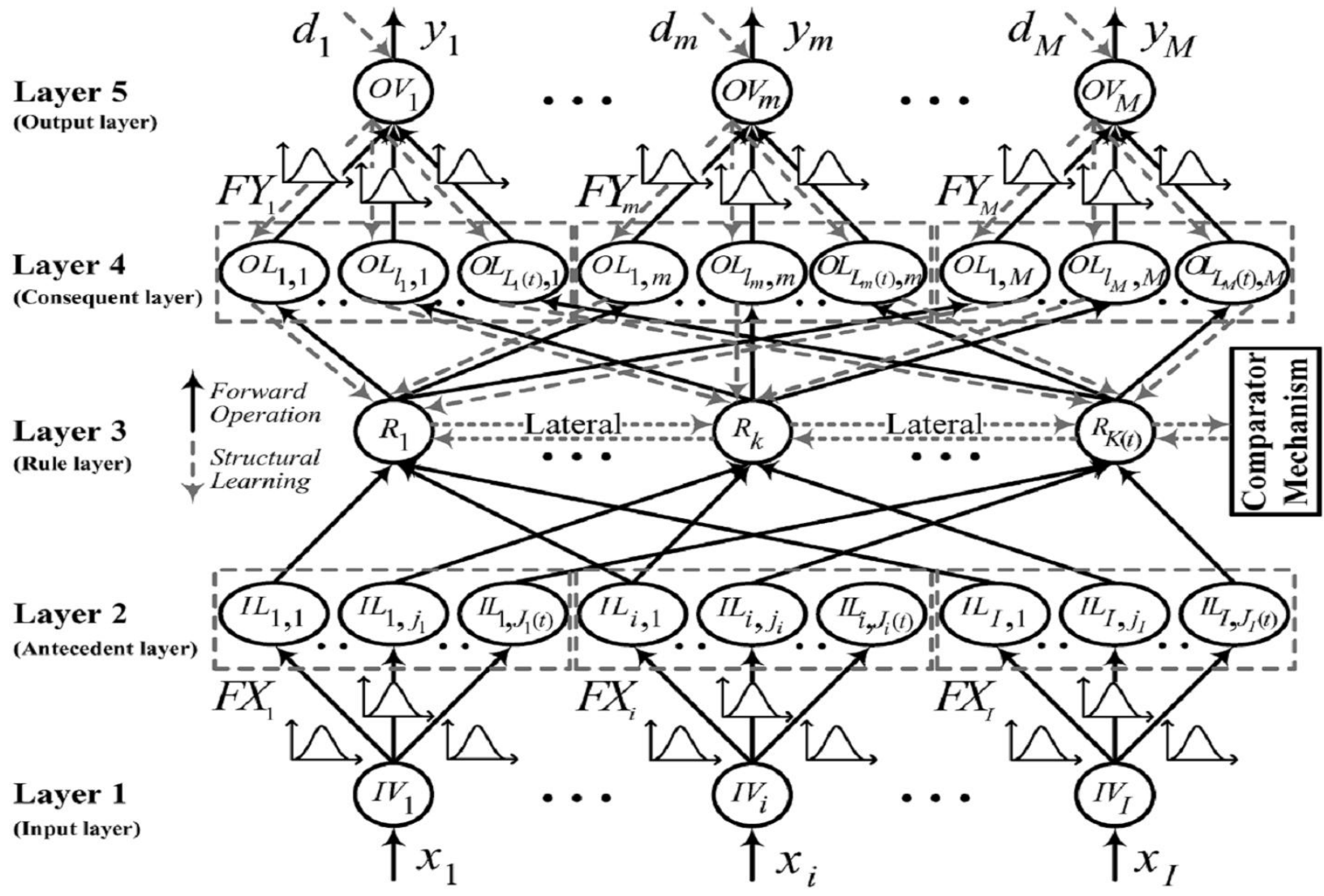

Figure 2. Computing structure of the eFSM neural-fuzzy semantic network [17]

The designed GUI also has several buttons that helps the user to decide what to do, these buttons are:

1. 'Form Question' button; to only display the formed question that may be correct or incorrect according to natural language.

2. 'Get One Answer' button; to both display the formed question, and estimate the single corresponding answer for this question.

3. 'Get Multi-Answers' button; to both display the formed question, and estimate the corresponding answer(s) for this question.

4. 'Read' button; is used to activate the editing textbox to insert the number, larger than 2 , of answer(s) that will be displayed.

The formed question may be correct and has answer(s) or may be incorrect with no answer. This depends on the chosen components form the input popup menus to form a meaningful question according to the natural language.

\subsection{The proposed System Layers}

In common, the design of neural-fuzzy system has five layers consists of two input/output layers and three hidden layers. This is illustrated in both Figure 2 and Figure 3.
For proposed system the layers are summarized as:

1. The input layer.

2. The input membership function layer.

3. The rules layer.

4. The output membership function layer.

5. The output layer.

Each layer will be discussed separately as follows:

\subsubsection{The input layer.}

This layer is described by the entered values that the user selected from the several popup menus. It has four elements discussed in details as in Table2. Each node represents an input variable to the FIS. The set of input layer nodes $I V_{i}(i=$ $1, \ldots, I)$ directly transposed to the input vector $X=$ $\left[x_{1}, \ldots, x_{i}, \ldots, x_{I}\right]^{T}$; where $\mathrm{I}$ is number of inputs; to the next layer as described in

$$
I V_{i}\left(x_{i}\right)=x_{i}, i=1, \ldots, I
$$

Where $I V_{i}\left(x_{i}\right)$ denotes the output of node $I V_{i}$ given input $x_{i}$. 
Table 2: The input layer's elements details

\begin{tabular}{|c|c|c|}
\hline Element & Values & Description \\
\hline Popup1 & $\begin{array}{l}\text { 'is', 'color is', 'can', and } \\
\text { 'does'. }\end{array}$ & $\begin{array}{l}\text { Defines the verb or } \\
\text { the auxiliary verb of } \\
\text { the question. }\end{array}$ \\
\hline Item & 'pine', 'oak', 'rose', , ..., etc. & $\begin{array}{lrr}\text { Defines } & \text { the } & \text { item } \\
\text { selected } & \text { in } & \text { the } \\
\text { question. } & & \end{array}$ \\
\hline Popup3 & '?', 'do?', and 'have?' & $\begin{array}{l}\text { Completes } \\
\text { question }\end{array}$ \\
\hline $\begin{array}{l}\text { Multi- } \\
\text { answer }\end{array}$ & $\begin{array}{l}\text { - ' ' } 1^{\text {st }} \text { answer/one } \\
\text { answer.' } \\
\text { - ' ' } 2^{\text {nd }} \text { answer/two } \\
\text { answers.' } \\
\text { - 'more ...' }\end{array}$ & $\begin{array}{l}\text { Defines the answer } \\
\text { and/or the number of } \\
\text { answers that will be } \\
\text { displayed. }\end{array}$ \\
\hline
\end{tabular}

\subsubsection{The input membership function layer.}

This layer is considered the first hidden layer. The input membership functions are represented as trapezoidal membership functions; 'trapmf' function. Each element of the four inputs is adjusted to be approximately pulse function.

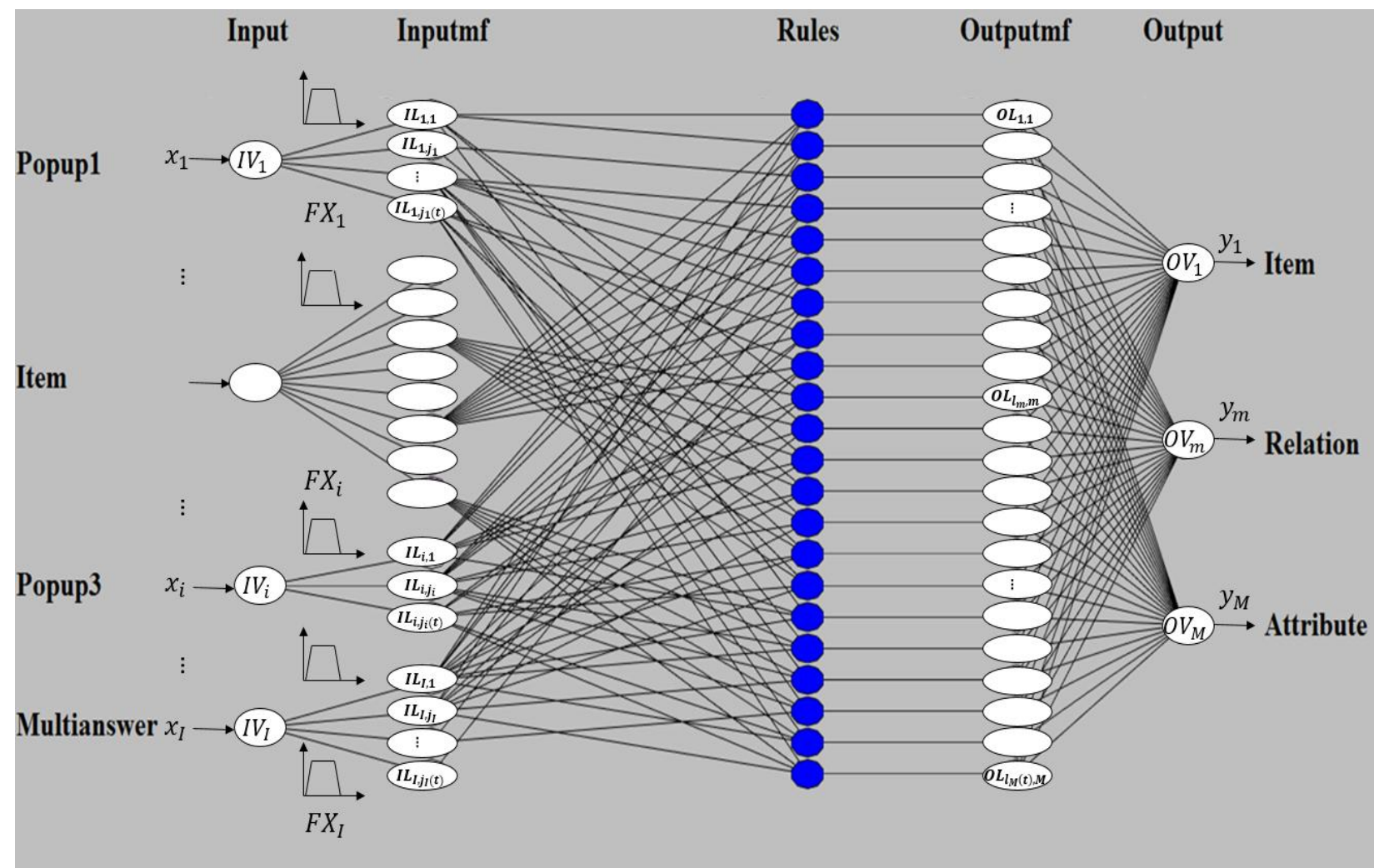

Figure 3. Neural-network for the proposed semantic memory model

This pulse function used here to avoid the overlapping that may happen in the selection of any element by the choice of the pulse intermediate value. Figure 4 illustrates the corresponding membership functions for the first popup menu 'popup1' and its different input variables with highlighted 'is' variable.

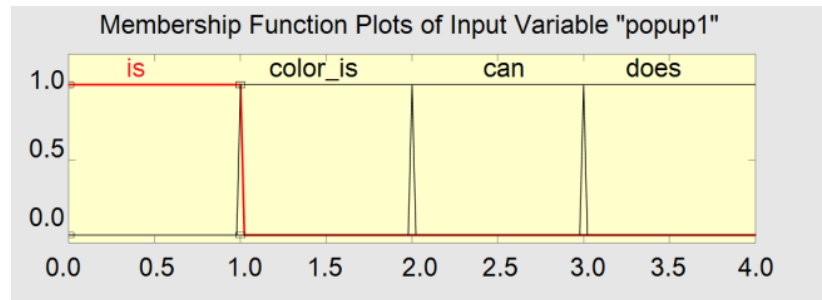

Figure 4 First popup menu membership function plots.

This layer holds the input fuzzy sets $I L_{i, j_{i}}[i=1, \ldots, I ; j=$ $\left.1, \ldots, J_{i}(t)\right]$ that define the antecedents of the Sugeno-type IF-THEN fuzzy rules generated by the FIS.F $X_{i}$ denotes the sets of fuzzy labels $\left\{I L_{i, j_{i}}\right\}_{j_{i}=1, \ldots, J_{i}(t)}$ that belongs to input 
variable $I V_{i}$ is represented by trapezoidal membership function. The membership value $I L_{i, j_{i}}\left(x_{i}\right)$ of input $x_{i}$ to the $j_{i}$ th fuzzy set of the associated input node $I V_{i}$ is given by

$I L_{i, j_{i}}\left(x_{i}\right)=f_{i, j_{i}}\left(x_{i} ; a_{i, j_{i}}, b_{i, j_{i}}, c_{i, j_{i}}, d_{i, j_{i}}\right), \quad i=1, \ldots, I, j_{i}=$ $1, \ldots, J_{i}(t)$

$$
I L_{i, j_{i}}\left(x_{i}\right)=\left\{\begin{aligned}
0, & x_{i} \leq a_{i, j_{i}} \\
\frac{x_{i}-a_{i, j_{i}}}{b_{i, j_{i}}-a_{i, j_{i}}}, & a_{i, j_{i}} \leq x_{i} \leq b_{i, j_{i}} \\
1, & b_{i, j_{i}} \leq x_{i} \leq c_{i, j_{i}} \\
\frac{d_{i, j_{i}}-x_{i}}{d_{i, j_{i}}-c_{i, j_{i}}}, & c_{i, j_{i}} \leq x_{i} \leq d_{i, j_{i}} \\
0, & d_{i, j_{i}} \leq x_{i}
\end{aligned}\right\}
$$

Where the parameters $a_{i, j_{i}}$ and $d_{i, j_{i}}$ locate the feet of the $j_{i}{ }^{\text {th }}$ trapezoid and the parameters $b_{i, j_{i}}$ and $c_{i, j_{i}}$ locate the shoulders.

Each value of the selected membership function input will be adjusted to be located in middle membership unction by a simple equation that decrease its value by 0.5 to locate in the center of pulse function.

\subsubsection{The rules layer.}

This is the second hidden layer. In this layer, the proposed system uses FIS structure to model the stored data of the semantic memory as a set of IF-THEN rules. It has four antecedent inputs and three outputs, shown in Figure 5. FIS rules control the relationship between the model inputs and outputs.

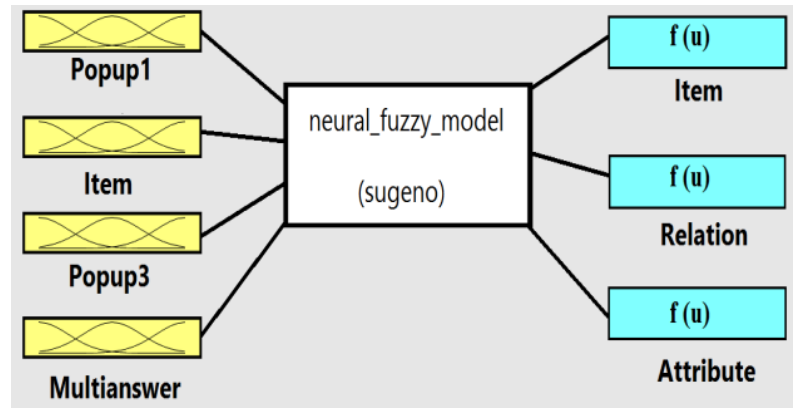

Figure 5. Trained FIS structure

To train new 'item' data and its' corresponding 'attributes', it is required to insert new training rules. The firing rule $R_{k}$ due to the activation of its antecedent section by the observed input $\mathrm{X}$ is computed as

$$
\begin{gathered}
R_{k}: I F \underbrace{\left(x_{1} i s I L_{1, j_{1}^{k}}\right) \ldots\left(x_{1} i s I L_{i, j_{i}^{k}}\right) \ldots\left(x_{1} i I L_{I, j_{l}^{k}}\right)}_{\text {Then }} \\
\underbrace{\left(y_{1} i s O L_{l_{1}^{k}, 1}\right) \ldots\left(y_{m} \text { is } O L_{l_{m}^{k}, m}\right) \ldots\left(y_{M} i s O L_{l_{M}^{k}, M}\right)}_{k=1, \ldots, k(t)} \\
R_{k}(X)=\min _{\mathrm{i}=1, \ldots, \mathrm{I}}\left(\left(\frac{x_{i}-a_{i, j_{i}}}{b_{i, j_{i}}-a_{i, j_{i}}}\right), 1,\left(\frac{d_{i, j_{i}}-x_{i}}{d_{i, j_{i}}-c_{i, j_{i}}}\right)\right), k= \\
1, \ldots, k(t)
\end{gathered}
$$

For examples the rule syntax used in simulation are:

- $\quad$ If $(($ popup $1=$ is $) \&($ item $=$ canary $) \&($ popup3 =?) \& $($ multianswer $=$ ans 1$))$ then $($ item $=$ canary $)($ relation $=$ isa) (attribute Rule 1

living_thing)

If $(($ popup $1=$ is $) \&($ item $=$ canary $) \&($ popup $3=$ ?) \& $($ multianswer $=$ ans 2$))$ then $($ item $=$ canary $)($ relation $=$ isa) (attribute $=$ bird) ........................................Rule 2

- If $(($ popup $1=$ color_is $) \&($ item $=$ canary $) \&($ popup3 $=$ ?) $\&($ multianswer $=$ ans 1$))$ then $($ item $=$ canary $)$ (relation $=$ is $) \quad($ attribute $=$ yellow $)$ ..................................Rule 3

There is a trained ' 80 ' different items with ' 133 ' attributes and connected via ' 528 ' relations that are represented by rule insertion in the proposed model. Each one of these data can be read from an external database to simplify the insertion of new item or feature.

\subsubsection{The output membership function layer.}

This layer is considered the third hidden layer, in which the relation of each item is related to its corresponding attributes. It has three output variables defined as Table3.

Table 3: The output membership function details

\begin{tabular}{||l|l|l||}
\hline Element & \multicolumn{1}{|c|}{ Value } & \multicolumn{1}{c|}{ Description } \\
\hline \multirow{2}{*}{ Item } & $\begin{array}{l}\text { 'pine', 'oak', 'rose', } \\
\text { 'daisy', 'robin', 'canary', } \\
\text { 'sunfish',... etc. }\end{array}$ & $\begin{array}{l}\text { Defines the selected item in } \\
\text { the question, similar to item } \\
\text { element of the input layer. }\end{array}$ \\
\hline Relation & $\begin{array}{l}\text { 'is_a', 'is_an', 'is', 'can', } \\
\text { and, 'has'. }\end{array}$ & $\begin{array}{l}\text { Is considered the verb of the } \\
\text { output statement. }\end{array}$ \\
\hline \multirow{2}{*}{ Attribute } & $\begin{array}{l}\text { 'living thing, plant, animal, } \\
\text { tree, flower, bird, green, } \\
\text { red, yellow, grow, ... etc. }\end{array}$ & $\begin{array}{l}\text { Represents the corresponding } \\
\text { attribute for the chosen item. }\end{array}$ \\
\hline
\end{tabular}

The output labels are organized according to the output variables $O V_{m}, m=1, \ldots, M, F Y_{m}$ denotes the set of output labels belonging to the output node $O V_{m}$.

The activation of $O L_{l_{m}, m}$ with respect to $\mathrm{X}$ is defined as

$$
\begin{gathered}
L_{l_{m}, m}(X)=\max _{\mathrm{R}_{\mathrm{k}} \in R_{l_{m}, m}}\left(R_{k}(X), 0\right), \\
l_{m}=1, \ldots, L_{m}(t), m=1, \ldots, M
\end{gathered}
$$

\subsubsection{The output layer (defuzzification layer).}

This layer integrates the FIS output membership functions to evaluate the corresponding output values. The function of an output node $O V_{m}$ is to combine the activations of all its input fuzzy sets in the previous layer to produce a response $y_{m}(X)$ to the input vector $X$. This is described in

$$
y_{m}(X)=\frac{\sum_{i=1}^{k} \omega_{i} O L_{l_{m}, m}(X)}{\sum_{i=1}^{k} \omega_{i}}, m=1, \ldots, M
$$

Where $\omega_{i}$ the firing strength of membership function.

$\omega_{i}=\operatorname{AndMethod}\left(I L_{1, j_{1}^{k}}, \ldots I L_{i, j_{i}^{k}}, \ldots I L_{I, j_{l}^{k}}\right) \quad$, the AndMethod is by Product method

Output vector $Y=\left[y_{1}, \ldots, y_{m}, \ldots, y_{M}\right]^{T}$; where $\mathrm{M}$ is number of outputs.

The output $\mathrm{Y}$ has integer values evaluated by the FIS and then it will be translated to corresponding word and displayed on GUI. 


\subsection{Answer probabilities}

The displayed answer(s) may be one of the following:

1. 'No answer(s) for wrong question', when the selected inputs forms meaningless question according to common natural language.

2. 'No answer is defined, it is needed to train data', when the question asked is correct but not included in the trained data of FIS structure.

3. If the trained data don't have a correct multianswer value it will result in an alert massage 'No more data is available, please select lower multianswer value.' and it is needed to choose less value from this menu.

4. The correct single answer or multi-answers according to the user choice.

\subsection{Practical Example:The question asked is 'what can canary do?':}

When 'Get Multi-Answers' button is pressed, and selected 'more ...' for third popup menu, and then press read with number 5 inserted so the corresponding steps will be applied to get the final answers:

1. The question input popup menu values are:

- $\quad$ Popup $1=3$ $\rightarrow \quad$ the verb 'can'.

- Item $=6 \quad \rightarrow \quad$ the item 'canary'.

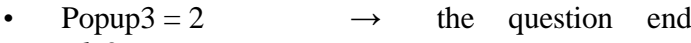
'do?'.

- Multi-answer $=3 \rightarrow \quad$ the selection of 'more ...'

2. So, the input vector will be ' 36223 ' with an inserted value ' 5 ' to the edit textbox as multi answer. This will result in a different input vector. This modification will be ' 3625 '

3. These values are adjusted to be '2.5 5.51 .54 .5 ' before being applied to FIS model, to avoid interference between the membership function input values. This step is recommended to avoid the overlapping between the input membership functions.

4. The program then calls the 'readfis' function that reads the FIS and load it to be able to use its features.

5. Then as the value of multi-answer $=5$ then there will be more than one step to evaluate the corresponding answers and display them all.

6. For the previous reason ,the program calls the function 'evalfis' more than one time to evaluate the corresponding output membership functions for the input pattern. This will be repeated three times and stored in three output variables

- $\quad$ Out1 = ‘6 4 10’.

- $\quad$ Out2 =`64 11',
- $\quad$ Out3 = ‘6 4 13’.

- $\quad$ Out4 = '6 4 14'

- $\quad$ Out5 = ‘6 497 ’

7. Respectively, these output vectors is then inversely transformed to form the answer words as follows:

- Out $1=` 64$ 10’.

- $6 \rightarrow$ 'canary' item

- $4 \rightarrow$ 'can' relation.

- $10 \rightarrow$ 'grow' attribute.

- Out2 = '6 4 11'

- $6 \rightarrow$ 'canary' item.

- $4 \rightarrow$ 'can' relation.

- $11 \rightarrow$ 'move' attribute

Similarly other outputs as;

- $\quad$ Out5 = ‘6 497 '.

- $6 \rightarrow$ 'canary' item.

- $4 \rightarrow$ 'can' relation

- $97 \rightarrow$ 'produce_eggs' attribute

8. So the displayed answer is as illustrated in Figure 6.

\section{CONCLUSION AND FUTURE RESEARCH}

For large amount of storage data, it is recommended to arrange the data as a multi-level semantic structure. This structure will be constructed internally as hierarchal structure in a FIS model. Hence, this model will save the items as a tree of data, e.g., for a 'canary' item it will be subdivided from 'bird' item, which is a branch of 'living thing item' and so on. This can be used as an intelligent, efficient and reliable search engine that returns the appropriate answer(s) for any question. Also it will be acceptable not only to use the search engine as a keywords but to semantically relate this keywords and try to answer the asked question directly.

As a conclusion, this paper introduces a novel design of the connectionist semantic memory model using FIS structure The proposed 'Neural-fuzzy Connectionist Semantic Memory Model' is implemented using the Fuzzy Logic Toolbox of the MATLAB software. Also, a GUI is depicted to facilitate the user search procedures, as it helps the user to easily form question and returns the appropriate answer(s). The results are quiet accurate and encouraging as a promising approach for the future direction.

\section{ACKNOWLEDGMENT}

This work has been supported by the Science and Technology Center of Excellence 'STCE' - the Ministry of Military Production. 


\begin{tabular}{|c|c|c|c|c|c|c|}
\hline A & \multicolumn{4}{|c|}{ gui_code } & \multirow[b]{2}{*}{ do? } & - \\
\hline What & can & $\checkmark$ & canary & $\checkmark$ & & \\
\hline Question & \multicolumn{6}{|c|}{ What can canary do? } \\
\hline Multi_Answers & \multicolumn{3}{|c|}{ more ... } & & & \\
\hline \multicolumn{6}{|c|}{ Press read and Enter the number value of Mutli_Answers $>2$} & \multirow[t]{3}{*}{ Read } \\
\hline Form Question & & Get On & & Get Mu & Answ & \\
\hline Answer(s) & \multicolumn{5}{|c|}{$\begin{array}{c}\text { canary can grow. } \\
\text { canary can move. } \\
\text { canary can fly. } \\
\text { canary can sing. } \\
\text { canary can produce_eggs. }\end{array}$} & \\
\hline
\end{tabular}

Figure 6 Multi-answers of question 'What can canary do?'.

\section{REFERENCES}

[1] Sheu, Yu, Ramamoorthy, Joshi, and Zadeh, Semantic Computing, Chapter1, Institute of Electrical and Electronics Engineers, 2010.

[2] Julian Szymańki, and Wlodizislaw Duch, Knowledge Representation and Acquisition for Large-Scale Semantic Memory, IEEE, 2008.

[3] Walter Kintsch, Modeling Semantic Memory, 2010.

[4] MF. Bonner and M. Grossman, Semantic Memory: Cognitive and Neuroanatomical Perspectives, Academic Press: Elsevier, 2015.

[5] Endel Tulving and Wayne Donaldson, Organization of Memory, Ch.10, Episodic and Semantic Memory, Academic Press New York and London, 1972.

[6] Michael N. Jones, Jon Willits, and Simon Dennis, Models of Semantic Memory, Ch. 11, 2015.

[7] Julian Szymańki, and Wlodizislaw Duch, Information retrieval with semantic memory model, ScienceDirect, Elsevier, 2012.

[8] W.L. Tung and C. Quek, An adaptive fuzzy semantic memory model based on the computational principles of the human hippocampus, IEEE, 2008.

[9] James L. Mcclelland, Connectionist Models of Memory, Chapter 36, The Oxford Handbook of Memory, 2000.

[10] Allan M. Collins and M. Ross Quillian, Retrieval time from semantic memory, Journal of Verbal Learning and Verbal Behavior, 1969.

[11] Timothy. T.Rogers, Computational models of semantic memory, Ch.8, The Cambridge Handbook of Computational Cognitive Modeling, 2008.

[12] Allan M. Collins, Elizabeth F. Loftus, A spreading activation theory of semantic processing, Psychological Review, 1975.

[13] David E. Rumelhart, Brain style computation learning and generalization, Chapter 21, Academic Press, 1990.
[14] Geoffrey E. Hinton, James A. Anderson, Parallel Models Of Associative Memory, 2014.

[15] David E. Rumelhart, Peter M. Todd, Learning and connectionist representations, chapter1, 1993.

[16] Timothy T. Rogers, James L. McClelland, Are theories necessary to constrain concepts?, the Cognitive Neuroscience Society, 2000.

[17] Tung And Quek, eFSM-A Novel Online Neural-Fuzzy Semantic Memory Model, 2010.

[18] W. L. Tung, and C. Quek, Brain-inspired fuzzy semantic memory model for learning and reasoning with uncertainty, 2007.

[19] Timothy T. Rogers and James L. McClelland, Précis of Semantic Cognition: A Parallel Distributed Processing Approach, 2008.

\section{AUTHOR PROFILE}

Noha M. Zakzouk, received the B.Sc. degree in Computer and Systems Engineering, Zagazig University, 2009. She Participated in MIE (Made in Egypt) 2009 Committee with graduation project and won the fourth rank. She works as instructor at the Ministry of Military Production, Training Sector, Cairo, Egypt.

Hassan M. Bedour, Ain Shams University, Faculty of Engineering, Computer and System Engineering Department. Ph.D. in 1990, University of Grenoble France - Ain Shams University. Research interests: computer architecture, system design, software architecture robotics.

Dina M. Khorshied, received the B.Sc. degree in electrical power and machines, Cairo University, Giza, Egypt, 1999 M.Sc. in electrical power and machines, Cairo University, Giza, Egypt, 2006, and Ph.D. in electrical power and machines, Cairo University, Giza, Egypt, 2012. She worked in the field of DC electric traction substations. Currently, she is working at Science and Technology Center of Excellence (STCE), Egypt. 